\title{
AJUSTE DE DADOS EXPERIMENTAIS DA SOLUBILIDADE DA UREIA EM SOLUÇÕES DE ISOPROPANOL+ÁGUA COM O USO DE EQUAÇÕES EMPÍRICAS
}

\author{
L. G. FONSECA ${ }^{1}$, J. B. RODRIGUES ${ }^{1}$ e R. A. MALAGONI ${ }^{1}$ \\ ${ }^{1}$ Universidade Federal de Uberlândia, Faculdade de Engenharia Química \\ E-mail para contato: malagoni@,feq.ufu.br
}

\begin{abstract}
RESUMO - A ureia é um composto orgânico envolvido em muitos processos biológicos e por isso seu comportamento em solução é um tópico importante em estudos ambientais. Neste trabalho foram feitos ajustes usando equações empíricas de diversos dados experimentais de solubilidade da ureia em diferentes concentrações e temperatura. O ajuste dos dados experimentais consistiu na determinação dos parâmetros das equações escolhidas juntamente com seus erros padrões e significância. Depois de definir os parâmetros, geraram-se as curvas de solubilidade em função da temperatura, o que tornou possível determinar a solubilidade em temperaturas não estudadas experimentalmente.
\end{abstract}

\section{INTRODUÇÃO}

A ureia é o fertilizante nitrogenado mais utilizado no mundo e também possui muitas aplicações industriais, incluindo fabricação de resinas, colas, solventes, medicamentos, produtos de limpeza e cosméticos (Francis et al., 2002). Ela é altamente solúvel em água e apresenta baixa solubilidade em solventes orgânicos não polares (Catchpole et al., 2005).

Dados de solubilidade são necessários em projetos de equipamentos de separação da indústria química. No entanto, a obtenção desses dados, em laboratório, geralmente despende tempo para agitação, repouso da solução e análise das amostras. Alguns artigos apresentam resultados de solubilidade para uma faixa de temperatura pequena e em poucas concentrações de solventes. O uso de equações empíricas para o ajuste de dados de solubilidade é uma alternativa, a qual possibilita estimar a solubilidade.

O objetivo deste trabalho foi avaliar a qualidade de ajustes feitos com equações empíricas para a solubilidade da ureia em função da temperatura. Os parâmetros para cada equação foram calculados e com o uso desses parâmetros gerou-se os gráficos utilizados nesse trabalho.

\section{MATERIAL E MÉTODOS}

\subsection{Procedimento Experimental}

A unidade experimental de solubilidade e os reagentes utilizados são os mesmos descritos em Silva et al. (2014). Em cada experimento foram utilizadas duas células de vidro borossilicato encamisadas que foram conectadas em série por uma mangueira de látex. Em 
cada uma das células colocavam-se as respectivas soluções de isopropanol+água na proporção em que se desejava analisar e ureia em excesso.

O período de agitação das soluções foi de $2 \mathrm{~h}$ seguido de $2 \mathrm{~h}$ de repouso. Após terminado o tempo de repouso foram retiradas quatro amostras de 4 a $5 \mathrm{~mL}$ de cada solução com o auxílio de uma seringa de vidro. Cada amostra foi retirada da célula e logo em seguida colocada no respectivo béquer para que sua pesagem fosse efetuada.

Após a pesagem do béquer com a amostra, os mesmos foram colocados na estufa (Marconi, MA-033), onde permaneceram por um período de $24 \mathrm{~h}$ na temperatura de 338,15 K. Após esse período, as amostras foram colocadas em um dessecador com sílica por 30 min e então foi feita a pesagem do béquer novamente para determinar a massa de ureia seca. Esse procedimento foi feito durante 4 dias até a massa ficar constante. Com a massa do béquer vazio, do béquer com a solução e do béquer com a ureia seca foi possível determinar a solubilidade para cada solução e em cada temperatura. O resultado de solubilidade obtido foi a média aritmética dos valores de solubilidade encontrados para cada amostra.

\subsection{Ajustes de Solubilidade}

Os ajustes utilizados neste trabalho foram feitos através de duas equações empíricas. A Equação 1 apresenta o modelo de Lee e Lahti (1972) e a Equação 2 representa o modelo de Yaws et al. (1993). A determinação dos parâmetros e a geração dos gráficos foram feitas usando o software Statistica.

$$
\begin{aligned}
& \ln S=A+B T \\
& \log S=A^{\prime}+\frac{B^{\prime}}{T}+\frac{C^{\prime}}{T^{2}}
\end{aligned}
$$

sendo que: $S$ é a solubilidade da ureia, $T$ é a temperatura em Kelvin, $A$ e $B$ são os parâmetros da equação de Lee e Lahti e $A^{\prime}, B^{\prime}$ e $C^{\prime}$ são os parâmetros da equação de Yaws et al.

Para avaliar a eficiência de cada ajuste utilizado foi efetuado o cálculo dos resíduos através da Equação 3.

$$
R=S_{\text {exp }}-S_{\text {calc }}
$$

\section{RESULTADOS E DISCUSSÃO}

\subsection{Solubilidade}

As Tabelas 1 e 2 apresentam os dados de solubilidade obtidos para as porcentagens gravimétricas de 10 e $40 \%$ de isopropanol m/m, respectivamente. 
Tabela 1 - Solubilidade da ureia em isopropanol+água (10\% isopropanol $\mathrm{m} / \mathrm{m}$ ).

\begin{tabular}{cc}
\hline$T \pm \delta(\mathrm{K})$ & $S \pm \delta(\mathrm{g}$ ureia/100g solvente $)$ \\
\hline $278,5 \pm 0,2$ & $66,1569 \pm 0,0186$ \\
$283,3 \pm 0,1$ & $79,1430 \pm 0,2430$ \\
$288,3 \pm 0,0$ & $88,4284 \pm 0,1930$ \\
$293,2 \pm 0,2$ & $98,1323 \pm 1,5126$ \\
$298,1 \pm 0,2$ & $103,6148 \pm 1,7900$ \\
$303,4 \pm 0,2$ & $125,9579 \pm 0,2386$ \\
$307,9 \pm 0,1$ & $140,0652 \pm 0,7371$ \\
$312,8 \pm 0,4$ & $153,1213 \pm 1,2367$ \\
$318,4 \pm 0,3$ & $161,1721 \pm 4,4737$ \\
$322,5 \pm 0,5$ & $185,1029 \pm 0,8107$ \\
$327,9 \pm 0,2$ & $192,8883 \pm 5,1605$ \\
$332,6 \pm 0,5$ & $226,7360 \pm 0,2805$ \\
\hline
\end{tabular}

Tabela 2 - Solubilidade da ureia em isopropanol+água ( $40 \%$ isopropanol $\mathrm{m} / \mathrm{m}$ ).

\begin{tabular}{cc}
\hline$T \pm \delta(\mathrm{K})$ & $S \pm \delta(\mathrm{g}$ ureia/100g solvente $)$ \\
\hline $278,2 \pm 0,1$ & $37,8215 \pm 0,2496$ \\
$283,2 \pm 0,0$ & $54,3019 \pm 0,0810$ \\
$293,1 \pm 0,1$ & $68,4791 \pm 0,2206$ \\
$298,2 \pm 0,2$ & $79,2912 \pm 0,1237$ \\
$303,4 \pm 0,2$ & $88,3213 \pm 0,2837$ \\
$307,7 \pm 0,1$ & $98,1705 \pm 0,8727$ \\
$313,1 \pm 0,4$ & $106,2550 \pm 4,8082$ \\
$318,4 \pm 0,3$ & $127,1020 \pm 0,2741$ \\
$322,7 \pm 0,2$ & $138,0328 \pm 0,7339$ \\
$328,0 \pm 0,2$ & $149,8612 \pm 4,2601$ \\
$333,0 \pm 0,1$ & $174,2320 \pm 3,8795$ \\
\hline
\end{tabular}

\subsection{Ajustes dos dados de solubilidade}

A Tabela 3 apresenta os parâmetros encontrados para as Equações (1) e (2) para a solução contendo $10 \%$ de isopropanol, juntamente com seus erros padrão e com o coeficiente de correlação quadrático. 
Tabela 3 - Parâmetros obtidos no ajuste de solubilidade (10\% isopropanol m/m).

Equação 1

Lee e Lahti (1972)
Equação 2

Yaws et al. (1993)

\begin{tabular}{cccc}
\hline Parâmetro & Erro Padrão & Parâmetro & Erro Padrão \\
\hline$A=-1,5583$ & 0,2379 & $A^{\prime}=5,3300$ & 2 \\
$B=0,0209$ & 0,0007 & $B^{\prime}=-1097,5100$ & 1216,7000 \\
- & - & $C^{\prime}=34884,8200$ & 187619,6000 \\
$\mathrm{R}^{2}=0,9945$ & & $\mathrm{R}^{2}=0,9953$ & \\
\hline
\end{tabular}

Para um nível de 5\% na análise estatística, ambos os parâmetros de Lee e Lahti (1972) foram significativos, enquanto que para um mesmo nível de significância, apenas o parâmetro $A$ ' do Yaws et al. foi significativo. Avaliando o coeficiente de correlação quadrático, os parâmetros obtidos e os erros padrão dos mesmos, pode-se concluir que o modelo de Lee e Lahti foi o que melhor representou o comportamento dos dados obtidos para a solução de $10 \%$ de isopropanol. A Figura 1 apresenta as curvas que foram obtidas para cada um dos modelos utilizados juntamente com os dados experimentos utilizados neste trabalho que estão representados na forma de pontos.

Figura 1 - Ajuste dos dados de solubilidade $(10 \% \mathrm{~m} / \mathrm{m}$ isopropanol $)$.

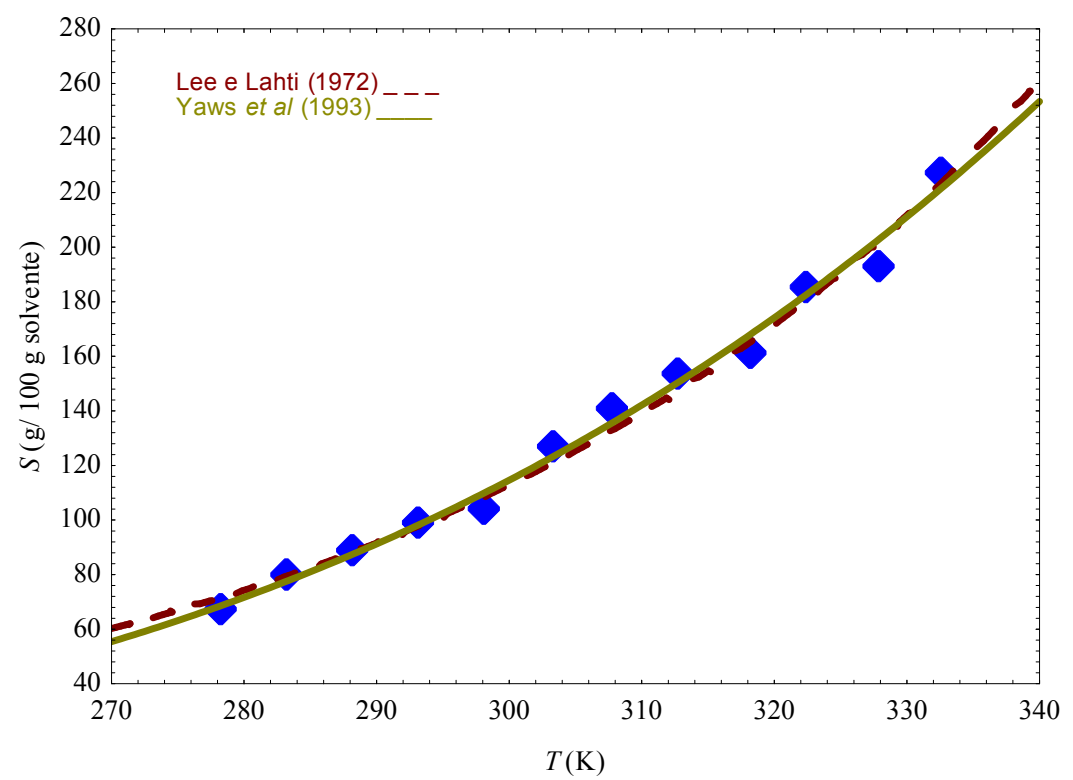

Os parâmetros encontrados para as Equações (1) e (2) para a solução contendo 40 \% de isopropanol estão reportados na Tabela 4, assim como seus erros padrão e o coeficiente de correlação quadrático para cada ajuste. Assim como no ajuste para a solução de $10 \%$ os parâmetros $A$ e $B$ de Lee e Lahti (1972) foram significativos enquanto apenas o parâmetro $A$ ' do Yaws et al. foi significativo. Novamente, o modelo de Lee e Lahti foi o que melhor representou o comportamento dos dados obtidos experimentalmente apresentando todos os parâmetros significativos e com baixos erros padrão. 
Tabela 4 - Parâmetros obtidos no ajuste de solubilidade (40\% isopropanol m/m)

Equação 1

Lee e Lahti (1972)
Equação 2

Yaws et al. (1993)

\begin{tabular}{cccc}
\hline Parâmetro & Erro Padrão & Parâmetro & Erro Padrão \\
\hline$A=-2,7481$ & 0,2729 & $A^{\prime}=4,7000$ & 1,9000 \\
$B=0,0237$ & 0,0009 & $B^{\prime}=-647,8000$ & 1189,1000 \\
- & - & $C^{\prime}=-54160,6000$ & 184319,3000 \\
$\mathrm{R}^{2}=0,9954$ & & $\mathrm{R}^{2}=0,9968$ & \\
\hline
\end{tabular}

A Figura 2 apresenta as curvas obtidas para cada modelo de ajuste utilizado e também os dados experimentais utilizados em cada ajuste. Pela figura é possível observar que a curva gerada pelo modelo de Lee e Lahti foi a que melhor ajustou os pontos obtidos experimentalmente. Os resíduos obtidos para o modelo de Lee e Lahti para a solução de 10\% $\mathrm{m} / \mathrm{m}$ de isopropanol foram em média iguais a 0,1034 e, para a de $40 \%$ de isopropanol, a média dos resíduos foi de 0,1641. Já para o modelo de Yaws et al., os resíduos obtidos tiveram média de 0,0064 para a solução de $10 \% \mathrm{~m} / \mathrm{m}$ de isopropanol e, de 0,1641 , para a solução de $40 \% \mathrm{~m} / \mathrm{m}$ de isopropanol.

Figura 2 - Ajuste dos dados de solubilidade (40\% $\mathrm{m} / \mathrm{m}$ isopropanol).

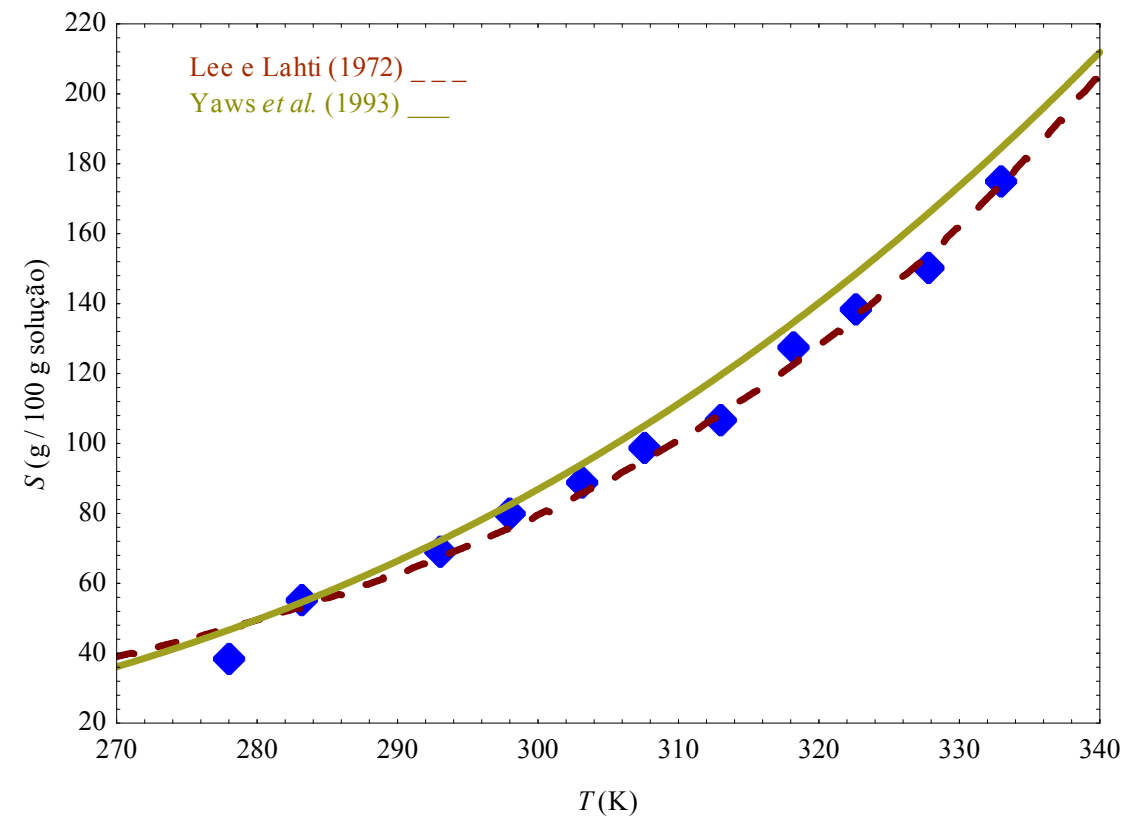

A Figura 3 mostra a relação entre a solubilidade experimental e a solubilidade calculada para cada um dos modelos utilizados e para as duas concentrações de isopropanol pesquisadas. 
Figura 3 - Relação entre $S_{\exp }$ e $S_{\text {calc }}$ para as soluções de isopropanol+água de 10 e $40 \%$.
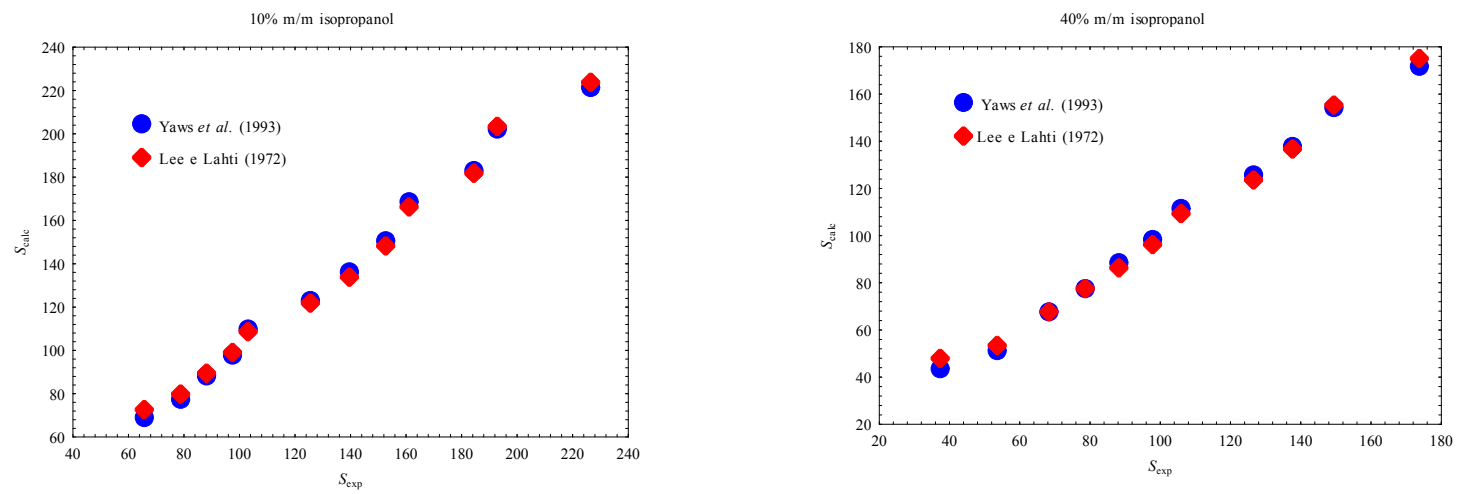

\section{CONCLUSÃO}

Para ambas as soluções, o modelo de Lee e Lahti foi o que melhor representou os dados obtidos experimentalmente tendo apresentado baixos erros padrão e todos os parâmetros significativos. Ele também apresentou baixos valores de resíduo, o que indica que os valores preditos pelo modelo ficaram próximos aos valores obtidos experimentalmente. Analisandose o coeficiente de correlação quadrático, é possível observar que o valor do mesmo é alto, o que comprova novamente a eficiência do modelo para ajustar os dados obtidos neste trabalho.

Para as misturas de solventes e faixa de temperatura estudadas neste trabalho o modelo de Yaws et al. não foi apropriado, pois apenas um de seus parâmetros mostrou-se significativo. Portanto, apesar deste modelo também ter apresentado baixos valores de resíduos e um bom coeficiente de correlação quadrático, os altos erros padrão dos parâmetros torna o modelo de Yaws et al. pouco indicado para analisar os dados experimentais desde trabalho.

\section{AGRADECIMENTOS}

Agradecemos a Fundação de Amparo à Pesquisa do Estado de Minas Gerais (FAPEMIG) pela bolsa de Iniciação Científica e também à Faculdade de Engenharia Química da Universidade Federal de Uberlândia pelo incentivo a pesquisa e estrutura física oferecida para o desenvolvimento desta pesquisa no Laboratório de Cristalização.

\section{REFERENCIAS}

CATCHPOLE, O. J.; TALLON, J. S.; DYER, J. P.; LAN, S.-J.; JENSEN, B.; RASMUSSEN, O.K.; GREY, J. B. Measurement and modelling of urea solubility in supercritical $\mathrm{CO}_{2}$ and $\mathrm{CO}_{2}+$ ethanol mixtures. Fluid Phase Equiib., v. 237, p. 212-218, 2005.

FRANCIS, P. S., LEWIS, S. W, LIM, K. F. Analytical methodology for the determination of urea: current practice and future trends. Trends Anal. Chem., v.21, p.389-400, 2002.

LEE, F. M.; LAHTI, L. E. Solubility of urea in water-alcohol mixtures. J. Chem. and Eng. Data, v. 17, p. 304-306, 1972.

SILVA, A. P.; ANDRADE, R. A. F.; MALAGONI, R. A. Ensaios de solubilidade da ureia em etanol+metanol e água+metanol. In: XX COBEQ - Congresso Brasileiro de Engenharia Química, Florianópolis - SC, p. 1-9, 2014.

YAWS, C. L.; LIN, X.; BU, L. The water solubility of naphtenes. Chem. Eng., v. 10, p. 122-123, 1993. 